\title{
A GENERALIZATION OF THE CONCEPT OF ABSOLUTE RETRACT
}

C. W. SAALFRANK

1. Introduction. In this paper the notion of absolute retract $[4 ; 5]$ is generalized for compact Hausdorff spaces to a new concept which we call absolute homotopy retract (AHR). This concept AHR is a topological invariant and is characterized in two ways by Theorems (3.4) and (3.6). An example (3.5) is given which shows that an AHR need not be an absolute retract (AR), nor even an absolute neighborhood retract (ANR) [5]. A product theorem (3.7) is given for AHR. Finally, a characterization theorem (4.3) is given for absolute retracts (AR). The word mapping will always mean continuous mapping.

2. Homotopy retraction and extension. Definition (2.1). Given the sets $A$ and $B$ such that $B C A$, we say that the mapping $f$ is a homotopy retraction ( $h$-retraction) of $A$ into $B$ provided $f: A \rightarrow B$ ( $f$ is mapping of $A$ into $B$ ) and $f \mid B \simeq i_{B}$ (restriction of $f$ to $B$ is homotopic to the identity mapping $i_{B}$ of $B$ onto $B$ ). The set $B$ is called a homotopy retract ( $h$-retract) of $A$.

In his definition of $h$-retraction, Bartholomay [1] required that $f$ be a mapping of $A$ onto $B$. However, it is necessary that $f$ be defined as a mapping of $A$ into $B$ as stated in definition (2.1) in order to obtain the theory as developed in this paper. Clearly if $B$ is a retract [2] of $A$, then $B$ is a homotopy retract of $A$. Homotopy extension of a mapping was defined by Bartholomay [1] as follows.

Definition (2.2). Given the sets $A$ and $B$ such that $B \subset A$, and a mapping $f: B \rightarrow Y$ (into), we say that the mapping $g$ is a homotopy extension ( $h$-extension) of the mapping $f$ over $A$ relative to $Y$ provided $g: A \rightarrow Y$ (into) and $g \mid B \simeq f$ (restriction of $g$ to $B$ is homotopic to $f$ ).

If $f: A \rightarrow Y$ and $g: A \rightarrow Y$ and $f \simeq g$, then for clarity we will sometimes write $f \simeq g: A \rightarrow Y$. The following well-known result [6, p. 3] will be needed.

Lemia (2.3). If $f_{1} \simeq f_{2}: X \rightarrow Y, g: Y \rightarrow W$ and $h: Z \rightarrow X$, then $g f_{1}$ $\simeq g f_{2}: X \rightarrow W$, and $f_{1} h \simeq f_{2} h: Z \rightarrow Y$.

Lemma (2.4). If $f: P \rightarrow A$ and $P \subset P_{1}$, and $f$ admits an h-extension $g$

Presented to the Society, January 25, 1961 under the title Homotopy retraction properties for compact Hausdorff spaces; received by the editors March 30, 1960 and April 8, 1960 and, in revised form, June 24, 1960. 
over $P_{1}$ relative to $A$, then $f$ admits an h-extension over $P_{1}$ relative to any $h$-retract $B$ of $A$ provided $f(P) \subset B$.

Proof. By (2.1) there is mapping $r: A \rightarrow B$ such that $r \mid B \simeq i_{B}: B \rightarrow B$. Since $f(P) \subset B$, we can regard $f$ as mapping $f: P \rightarrow B$. By (2.3) therefore $i_{B} f \simeq(r \mid B) f: P \rightarrow B$ or $f \simeq r f: P \rightarrow B$. Now (2.2) implies $(g \mid P)$ $\simeq f: P \rightarrow A$. Using (2.3) we have $r(g \mid P) \simeq r f: P \rightarrow B$. By transitivity, $(r g) \mid P \simeq f: P \rightarrow B$. Since $r g: P_{1} \rightarrow B$, we have $r g$ is $h$-extension of $f$ over $P_{1}$ relative to $B$.

3. Absolute homotopy retract. Definition (3.1). A compact Hausdorff space $X$ is said to be an absolute homotopy retract (AHR) provided every topological image $X_{1}$ of $X$, which is a subset of a compact Hausdorff space $Y$, is an $h$-retract of $Y$.

From (3.1) two immediate results follow.

THEOREM (3.2). The property of being an AHR is a topological invariant.

TheOREm (3.3). Any compact absolute retract $[4 ; 5]$ is an $A H R$.

TheOREM (3.4). A necessary and sufficient condition for a space $A$ to be an $A H R$ is that $A$ be homeomorphic to a closed h-retract of some compact parallelotope $Q[3]$.

Proof. Necessity. Since $A$ is a compact Hausdorff space, by the Tychonoff imbedding theorem [3, p. 29], there exists a homeomorphism $h: A \rightarrow A_{1}$ which maps $A$ onto a subset $A_{1}$ of a compact parallelotope $Q$. Since $Q$ is a compact Hausdorff space [3, p. 29], by (3.1) we have $A_{1}$ is an $h$-retract of $Q$. The continuity of $h$ and compactness of $A$ imply $A_{1}$ is compact and therefore closed in $Q$.

SufFICIENCY. Let $h$ be a homeomorphism such that $h(A)=A_{1}$ where $A_{1}$ is closed $h$-retract of some compact parallelotope $Q$. Consider any other topological image of $A$, say $k(A)=A_{2}$ where $A_{2}$ is subset of compact Hausdorff space $Y$. $A_{1}$ closed in $Q$ implies $A_{1}$ compact and hence $A$ is compact Hausdorff space and $A_{2}$ is compact and hence closed in $Y$. Since $h k^{-1}: A_{2} \rightarrow Q$ where $Q$ is compact parallelotope, we apply Tietze's extension theorem [4, p. 1052, proof of Theorem (3.2)] and obtain extension $f$ of $h k^{-1}$ over $Y$ relative to $Q$. Now $A_{1}$ is $h$ retract of $Q$ implies there exists a mapping $g: Q \rightarrow A_{1}$ such that $g \mid A_{1} \simeq i: A_{1} \rightarrow A_{1}$. Therefore we have mapping $H: A_{1} \times(0,1) \rightarrow A_{1}$ surh that $H\left(a_{1}, 0\right)=g\left(a_{1}\right)$ and $H\left(a_{1}, 1\right)=a_{1}$. Define mapping $r: Y \rightarrow A_{2}$ by $r(y)=k h^{-1} g f(y)$ for $y \in Y$. Define mapping $K: A_{2} \times(0,1) \rightarrow A_{2}$ by $K\left(a_{2}, t\right)=k h^{-1} H\left(f\left(a_{2}\right), t\right)$ for $a_{2} \in A_{2}$. Since $f$ is extension of $h k^{-1}$, we 
have $f\left(a_{2}\right) \in A_{1}$ for $a_{2} \in A_{2}$ showing $K$ is well defined. Clearly $K\left(a_{2}, 0\right)$ $=r\left(a_{2}\right)$ and $K\left(a_{2}, 1\right)=a_{2}$. Therefore $A$ is an AHR.

EXAMPLe (3.5). We give an example of a compact Hausdorff space $A$ which is an AHR, but which is not an absolute retract [5] nor an absolute neighborhood retract [5]. Consider the 2-dimensional compact parallelotope $Q_{2}$, namely all $(x, y)$ such that $0 \leqq x \leqq 1$ and $0 \leqq y \leqq 1$. Let $A$ be the compact subset of $Q_{2}$ whose points are all $(x, y)$ such that $0 \leqq x \leqq 1$ and $y=x / n$ where $n=1,2,3, \cdots$ plus points $(x, 0), 0 \leqq x \leqq 1 . A$ not being locally connected at points $(x, 0)$, $0<x \leqq 1$, implies $A$ is not absolute retract or absolute neighborhood retract [5, Corollary 8.3]. Define mapping $f: Q_{2} \rightarrow A$ by $f\left(Q_{2}\right)=(0,0)$. Clearly $f \mid A \simeq i_{A}$ because of mapping $H: A \times(0,1) \rightarrow A$ defined by $H(x, y, t)=(t x, t y, 0)$. By (3.4) $A$ is an AHR.

In view of (3.3) and (3.5) the concept AHR is a generalization of the concept of absolute retract.

THEOREM (3.6). A necessary and sufficient condition for a set $A$ to be an $A H R$ is that $A$ be a compact Hausdorff space and that any mapping $f$ defined on a closed subset $P$ of a compact Hausdorff space $P_{1}$ such that $f: P \rightarrow A$, admits an $h$-extension over $P_{1}$ relative to $A$.

Proof. Necessity. By (3.4) $A$ is homeomorphic to a closed $h$ retract, say $h(A)=A_{1}$ of some compact parallelotope $Q$. Apply Tietze's extension theorem [4] to mapping $h f: P \rightarrow Q$ and obtain extension $g$ of $h f$ over $P_{1}$ relative to $Q$. Clearly $g$ is also an $h$-extension of $h f$ over $P_{1}$ relative to $Q$. Since $h f(P) \subset A_{1}$, apply (2.4) and obtain $h$ extension $k$ of $h f$ over $P_{1}$ relative to $A_{1} \cdot h^{-1} k$ is $h$-extension of $f$ over $P_{1}$ relative to $A$ because $k \mid P \simeq h f: P \rightarrow A_{1}$ and $h^{-1}: A_{1} \rightarrow A$ implies by (2.3) that $\left(h^{-1} k\right) \mid P \simeq f: P \rightarrow A$.

Sufficiency. Consider any topological image $h(A)=P$ such that $P$ is subset of compact Hausdorff space $P_{1}$. We will show $P$ is $h$ retract of $P_{1} . A$ is compact implies $h(A)=P$ is closed subset of $P_{1}$. Since $h^{-1}: P \rightarrow A$, there exists by hypothesis an $h$-extension $g$ of $h^{-1}$ over $P_{1}$ relative to $A$. The mapping $h g: P_{1} \rightarrow P$ is an $h$-retraction of $P_{1}$ into $P$ because $g \mid P \simeq h^{-1}: P \rightarrow A$ and $h: A \rightarrow P$ imply by (2.3) that $(h g) \mid P \simeq i_{P}$. By (3.1) $A$ is an AHR.

Theorem (3.7). If $\left\{A_{\sigma}\right\}, \sigma \in \Sigma$, is a collection of $A H R$, then the topological product $\prod A_{\sigma}$ is an $A H R$.

Proof. $A_{\sigma}$ is compact Hausdorff space for each $\sigma \in \Sigma$ implies $\prod A_{\sigma}$ is compact Hausdorff space [3]. Consider any closed subset $P$ of a compact Hausdorff space $P_{1}$ and any mapping $f: P \rightarrow \prod A_{\sigma}$. For $p \in P$, $f(p)=\left\{f_{\sigma}(p)\right\}$, where $f_{\sigma}(p) \in A_{\sigma}$ for each $\sigma \in \Sigma$. Therefore $f_{\sigma}$ is map- 
ping $f_{\sigma}: P \rightarrow A_{\sigma}$ for each $\sigma \in \Sigma$. By (3.6) (necessity) there exists $h$ extension $g_{\sigma}$ of $f_{\sigma}$ over $P_{1}$ relative to $A_{\sigma}$ for each $\sigma \in \Sigma$. Define mapping $G: P_{1} \rightarrow \prod A_{\sigma}$ by $G(p)=\left\{g_{\sigma}(p)\right\}$, for $p \in P_{1} . g_{\sigma} \mid P \simeq f_{\sigma}: P \rightarrow A_{\sigma}$ for each $\sigma \in \Sigma$ implies there exists a mapping $h_{\sigma}: P \times(0,1) \rightarrow A_{\sigma}$ for each $\sigma \in \Sigma$ such that $h_{\sigma}(p, 0)=g_{\sigma}(p)$ and $h_{\sigma}(p, 1)=f_{\sigma}(p)$. Define mapping $H: P \times(0,1) \rightarrow \prod A_{\sigma}$ by $H(p, t)=\left\{h_{\sigma}(p, t)\right\}$. Clearly $H(p, 0)=G(p)$ and $H(p, 1)=f(p)$. Therefore $G \mid P \simeq f: P \rightarrow \prod A_{\sigma}$. By (3.6) (sufficiency) $\prod A_{\sigma}$ is an AHR.

4. Characterization theorem for absolute retracts. We shall need the following two known results.

THEOREM (4.1). A necessary and sufficient condition for a space to be an absolute retract $(A R)$ is that it be homeomorphic to a retract of some compact parallelotope [5, Theorem 3.6].

Theorem (4.2) (Borsuk's THEOREM For COMPACT HaUSDORFF SPACES). Let $C$ be a closed subset of a compact Hausdorff space $X$, let $N$ be an $A N R$ and let $f \simeq g: C \rightarrow N$ ( $f$ and $g$ are homotopic mappings of $C$ into $N)$. Then if there is an extension $F$ of $f$ over $X$ relative to $N$, there is also an extension $G$ of $g$ over $X$ relative to $N$ with $F \simeq G: X \rightarrow N[5$, Theorem 6.4].

We can now prove the following characterization theorem for absolute retracts (compact Hausdorff).

Theorem (4.3). A necessary and sufficient condition for a space $A$ to be an absolute retract $(A R)$ is that $A$ be both an absolute neighborhood retract $(A N R)$ and an absolute homotopy retract $(A H R)$.

Proof. Sufficiency. Since the space $A$ is an AHR, we apply Theorem (3.4) and obtain a homeomorphism, say $h(A)=B$ where $B$ is a closed $h$-retract of some compact parallelotope $Q$. Hence there exists an $h$-retraction, say $f: Q \rightarrow B$ (into), such that $f \mid B \simeq i_{B}: B \rightarrow B$, where the mapping $f \mid B$ is the restriction of the mapping $f$ to $B$ and $i_{B}$ is the identity mapping on $B$. The property of being an ANR is a topollogical invariant [5, Theorem 3.8] and therefore $B$ is an ANR. Since the mapping $f$ is an extension of the mapping $f \mid B$ over $Q$ relative to $B$, by Theorem (4.2) there exists an extension $r$ of $i_{B}$ over $Q$ relative to $B$. Clearly the mapping $r(Q)=B$ is a retraction of $Q$ onto $B$. Hence by Theorem (4.1) we have $A$ is an AR.

Necessity. Since the space $A$ is an AR we apply Theorem (4.1), and obtain a homeomorphism, say $h(A)=B$ where $B$ is a retract of some compact parallelotope $Q$. By [5, Theorem 2.2], it follows that $B$ is a closed subset of $Q$. Since a retract of any space is clearly both a 
neighborhood retract and an $h$-retract of that space, it follows that $B$ is both a closed neighborhood retract and a closed $h$-retract of the compact parallelotope $Q$. Hence by [5, Theorem (3.4)] and Theorem (3.4), we have $B$ is both an ANR and an AHR.

It follows from Theorem (4.3) that any AHR which is not an AR cannot be an ANR. Example (3.5) in this paper is an example of such a space which is not locally connected. It is now natural to ask if there exist locally connected AHR (compact Hausdorff) which are not AR (compact Hausdorff). This question remains open.

\section{REFERENCES}

1. A. F. Bartholomay, Type-invariance and h-retraction, Portugal. Math. vol. 13 (1954) pp. 105-110.

2. K. Borsuk, Sur les rêtractes, Fund. Math. vol. 17 (1931) pp. 152-170.

3. S. Lefschetz, Algebraic topology, Amer. Math. Soc. Colloquium Publications, vol. $27,1942$.

4. S. T. Hu, A new generalization of Borsuk's theory of retracts, Nederl. Akad. Wetensch. Proc. vol. 50 (1947) pp. 1051-1055.

5. C. W. Saalfrank, Retraction properties for normal Hausdorff spaces, Fund. Math. vol. 36 (1949) pp. 93-198.

6. P. J. Hilton, An introduction to homotopy theory, Cambridge University Press, 1953.

Lafayette College 